\title{
「不能VR」と「V不R」
}

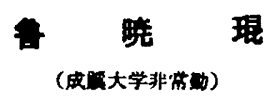

$\lceil\mathrm{V}$ 不 $\mathrm{R} 」 は$ 可能補語の否定式であり，「不能V R」は動詞と結果補話または 方向補語の前飞“不能”を加えたるのである。可能補語の肯定式「V得R」は多 数の情㫛の下で「能VR」で胃き奥えることができる。これは「能VR」も可能 の意味を示すからでる。一部の「V得R」が能VR」K贯导換えられないの は文法棬造に原因がある。しかし「V不R」は注んの一部の情況の下で「不能 VR」で贯きえることができる。そのはかの「V不R」は「不能VR」と枟換

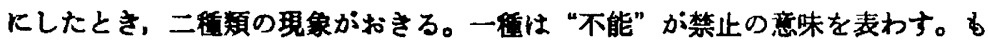
ら一種は転换されたあと，「不能VR」が成立できなくなる。上記の三つの現象

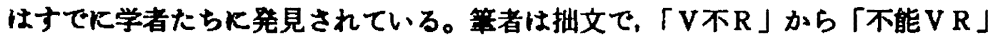
に転換して形成された上記の三つの現象を迥ってその形成の原因と規則を試みに 深りたいのである。即ち、「不比V R」の“不能”はどんな条件の下で可能の意 味を示し、どんな年件の下で禁止の意味を示し，どんな条件の下で意味がないか といらことを解明したいと思ら。

0. 前言

1.「不能VR」替换「V不R」表示可 能意义的条件

1.1.「不能V结」替换「V不结」表 示可能意义的疌件

1.2.「不能V趋」替换「V不趋」表 示可能意义的条件

1.3.「不能VR」替换「V不 $R$ 」表 示可能意义的条件

\section{0. 前言}

动结式和动趋式中间加上 “得” 或 “不” 就构 成了可能补语的肯定式和否定式, 我用「V 得 $R$ 」和「V不 $R$ 」表示。动结式和动趋式前 加 “能”或 “不能”, 我们用「能VR」和「不 能 V R」表示。

关于「能 V R」和「V得 $R 」$ 的关系，人们 探讨得比较多。多数「V得 $R J$ 都可以用「能
2.「V不R」变抰成「不能VR」后的 两种情况产生的原因

2.1.「不能VR」和「V不 $R 」$ 的否 定作用点不闰

2.2.「VR」结构的内部联系

2.3.「V不 $R\rfloor$ 变换成「不能 VR」 后的两种请况产生的原因

3. 结尾

V R」代替, 从而发现二者表达的意义基本相 同。但是由于结构形式的不同，二者出现的环 境仍有若干差别。

然而「不能 V R」和「V不 $R$ 」的关系却不 能与「能 V R」和「V得 $R 」$ 的关系相对称。 表现在形式上就是 $\lceil\mathrm{V}$ 不 $\mathrm{R} 」$ 在大多数情况下 不能用「不能V R」替换。但是人们也发现了 $\lceil V$ 不 $R 」$ 可以用「不能 V R 」替换的若干例 
文。住 例如：

(1)如果信巫不信医, 病是治不好的。一不 能治好

(2)你就是把他们的粮食捜光，也钱不掉他们

一颗大牙。 $\longrightarrow$ 不能饿掉

(3)眼泪喂不饱弟々。—不能喂饱

(4)……别说打不败官军和乡勇。一不能打 败

(5)在沙漠里养不出牡丹来。一不能养出来 上面五例划线部分的「V不 $R 」$ 都可用「不 能V R」替换。那么，什么条件下〔不能V R」 可以替换「V不 $R 」$ 表达可能的意义，还是一 个疑点，本文试图探讨这个问题。

在「不能VR」不能代替「V不 $R$ 」表示可 能意义的范围内, 「V不 $R 」$ 如果变换成「不 能VR」有两种情况。

一种是变换后句子就不成立了。如 :

(1)这本书我看不借。（*这本书我不能看㩐。）

(2)人太多，我买不上。（*人太多，我不能 买上。)

(3)我看不出来小张的才能。（*我不能看出 小张的才能。)

(4)这座山太高, 我把不上去。（*这座山太 高, 我不能爬上去。)

另一种是变换后句子仍然成立, 但 “不能” 表达的不是 “不可能”，而是 “禁止”的意义。 如 :

(1)黑板上的字徖不掉。（○黑板上的字不能 擦掉。)

(2)这些菜实不光。(

(3)吸烟的害处说不出来。(口吸烟的害处不 能说出来。)

(4)病人站不起来。( 病人不能站起来。)

「V不R」变换成「不能V R」后的两种形 式形成的原因是什么? 如何加以区别? 也是本 文力求解决的问题。

1.「不能VR」替换「V不 $R 」$ 表示可能 意义的条件

1.1.「不能 V结」替换「V不结」表示可
能意义的条件

根据目前的研究成果，划分结果补语的种类， 可以按照结果补语与句中不同成分构成的陈还 关系来划分。具有代表性的例句是：

(44)我们打完了球。(A类)

(15)我们打偗了球。(B类)

(16)我们打破了球。( $\mathrm{C}$ 类)

例(4)结果补语 “完” 是表示 “打” 这个动作 完了, A类是结果补语和谓语构成陈述关系; 例15结果补语 “面”是说 “我们軼了”, B类 是结果补语和句中施事构成陈述关系; 例16结 果补语 “破”是说“球破了”，C类是结果补 语和句中受事构成陈述关系。

A， B，C三类加上 “不” 都可构成可能补 语的「V不 $\mathrm{R}$ 。

(1D)我们打不完球。

(18)我们打不貸球。

(19)我们打不破球。

我们把这三类句式的「V不 $R 」$ 用「不能 $\mathrm{V}$ $\mathrm{R}$ 」替换一下, 就会发现下面的情况。

Q*我们不能打完球。

(21) *我们不能打栄球。

(20)我们不能打破球。

这三例表明， A类， B 类动结式构成的 $\Gamma \mathrm{V}$ 不结」不能用「不能V结」替换。C类动结式 构成的「V不结」可以用「不能 V 结」替换。 让我们再观察一些例文。
A 类《*那儿太远，我不能看清楚。
(24) * 那块石头重, 我不能投动。
(2)*在这里不能住上自己的房子。
B 类 * 我不能听借老师的课。
(2) * 我们去那儿, 不能玩痛快。
* 那里太吵, 不能住舒服。
C 类20我不能逗笑孩子。
300 孩子不能弄坏录音机。
(11) 这个工作不能累死人。

在不改变「V不结」意义的前提下，只有 $\mathrm{C}$ 类可用「不能 V 结」替换, 即「不能 V结」替 换「V不结」的条件是结果补语和句中受劣存 在着一种陈还关系。 
但是, 就 C类动结式, 我们所举出的例句句 中都有施事主语出现，如果把施素主语换成受 事主语, 就会出现不同的情况。如：

3○这种球打不破。

*这种球不能打破。

3○黑板上的字察不掉。

*黑板上的字不能擦掉。

3○他的病治不好。

*他的病不能治好。

这三例、「V不结」变换成「不能V结」后, 「不能V结」不表达可能的意义。

这种情况又进一步揭示，C类动结式构成的

「不能 V结」, 如果句中没有施亨主语出现， 也仍然不能表达可能的意义。

这是因为句中有没有施事主语出现「V不 结」中的“结果” 不能实现的原因不同。请看 下面的对比。

(39)他的病这个医生治不好。（不能治好）

3 他的病治不好。（*不能治好）

例39 “治不好”的原因在这个医生，例3 “治不好”的原因在病自身，说明他的病是不 治之应。也就是说在 $C$ 类动结式的「 $\mathrm{V}$ 不结」 句子中, 如果有施事出现, “结果”不能实现 的原因在施事，如果没有施事出现，“结果” 不能实现的原因在受本本身。

最后，我们把「不能V结」可替换「V不结」 表达可能意义的条件概括为 :

在 C类动结式构成的「 $\mathrm{V}$ 不结」的句子中, 如果句中有施事出现来表示「V不结」的原因, 这时，「V不结」可用「不能 V结」替换，反 过来说，「不能 V结」可以替换「V不结」表 达可能。

可以用「不能V结」的句子都可以变换成 “把”字句。如：

(32) 这个医生不能把他的病治好。

3 孩子不能把录音机弄坏。

(9)我们不能把球打破。

1.2.「不能V㭷」替换「V不趋」表示可 能意义的条件

在研究中我们发现, 划分结果补语种类的方
法也可以用在趋向补语上。即趋向补语也可与 句中的施高，受事形成陈述关系。如：

(40)他走回家去了。(A类)

(11)他寄回钱去了。(B类)

例49趋向补语“回去”是表示“他回去”, A 类趋向补语和句中施京构成陈述关系; 例 趋向补语 “回去”是“钱回去”，B类趋向补 语和句中受军构成陈述关系。

A， B 类都可加 “不” 构成可能补语「V不 趋」。

(42)他走不回家去。

(4) 他奇不回钱去。

我们把这两类句式的「V不急」试图用「不 能V趋」替换一下，就会发现下面的情况。

44*他不能走回家去。

(450他不能寄回钱去。

这两例表明， $\mathrm{A}$ 类动趋式构成的「V不趋」 不能用「不能 V趋」替换，B类动趋式构成的 「V不趋」可以用「不能V趋」替换。让我们 再观察两组例句 :

(16)他不能写出论文来。

*他不能走出房间来。

(10)他不能买出去这么多货。

*他不能跑出院子去。

再看下面分散的用例 :

A 类18*这座山太高，我不能爬上去。

9 *洞太小，孩子也不能钻进去。

$\mathrm{B}$ 类引○我不能搜集上来群众的意见。

610孩子不能搬进这么大的案子来。

在不改变「V不趋」意义的前提下，只有 B 类可用「不能 V趋」替换，即「不能 V 趋」替 换「V不趋」的条件是趋向补语和句中受事存 在着一种陈述关系。

但是, 象 C类动结式句一样， B 类动趋式我 们前面所举例句也都是句中有施事主语出现的， 如果去掉施每主语，「不能V趋」的“不能” 仍不能表达可能的意义。例如：

220钱寄不回去。

* 钱不能寄回去。

3○论文写不出来。 
*论文不能写出来。

90 这么多货实不出去。

*这么多货不能卖出去。

这种情况又进一步表明, B 类动趋式构成的 「不能V趋」，如果句中没有施主主语出现，也 仍不能表达可能的意义。原因和「不能V结」 的情况一样。

在 B 类「V不趋」句中, 如果有施事出现, “趋向”不能实现的原因在施事，如果没有施 事出现，“趋向”不能实现的原因在受亭本身， 或其他方面。

（2）他寄不回钱去。(O他不能寄钱去。）

36 钱寄不回去。(*钱不能寄回去。)

例囚奇不回钱去的原因在 “他”, 也许因为 “他”收入少, 也许因为 “他”花销大。例 “奇不回去”的原因一定在其他方面，如蚛局 不给奇等。

最后，我们把「不能 V趋」可替换「V不趋」 表达可能的条件櫭括为：

在 B 类动趋式构成的「V 不趋」的句子中， 如果句中有施事出现来表示「V不趋」的原因， 这时，「V不趋」可用「不能V趋」替换，反 过来说「不能 V趋」可以替换「V不趋」表达 可能。

1.3.「不能 VR」能替换「V不 R」表示 可能的条件

通过 1.1 和 1.2 的分析, 我们发现「不能 V 结」和「不能 V趋」的出现有相同的余件, 从 中可以概括出「不能VR」替换「V不R」表 达可能的年件。「不能 V R」替换「V 不 R」 表达可能必须具备下面两个条件。

(1) 「VR」中的 “ $R$ ” 要与句中受亭构成一 种陈述关系。

(2)在「不能 VR」出现的句子中，必须有施 事出现，表达 “不可能”的原因。

用这个结论我们基本上可以解释文章开头举 出的学者们发现的「V不 $R$ 可以用「不能V $R$ 」替换的若干例句。

(1)如果信巫不信医，病是治不好的。一不 能治好。
这个复句表达的是“巫是治不好病的”。句 中的结果补语 “好” 和受事”病”可以形成陈 述关系, 即 “病好”。其中 “巫” 是句中施事, 是治不好的原因。

(2)你就是把他们的粮食捜光, 也钱不掉他们

一颗大牙。一不能饿掉

这个复句可以压缩为 “你饿不掉他们一颗大 牙”。句中结果补语 “掉” 和受事 “大牙” 可以 形成一种陈述关系，即 “大牙掉”。其中 “你” 是句中施事，是“饿不掉”（大牙）的原因。

(3)眼泪喂不饱弟々。一不能喂饱

这一例最直接, “饱”与 “弟々”可以形成 陈达关系，“眼泪”是 “喂不饱”的原因。

(4)……别说打不败官军和乡要…… 一不能 打败

这个句中, “散”与“官军和乡勇”可以形成 一种陈述关系。问题是没有施事出现, 这是因 为有上文, 施事省略了, 我们可以补出施事。 说成 “他们别说…...”。

(5)在沙漠里养不出往丹来。一不能养出来 这个句中, 趋向动词 “出来”和句中受荸“任 丹” 可以形成一种陈述关系, 即“㤌丹出来”。 但是在句中没有施事, 代替施事表示“并不出 来”的原因的是句中状语。对状出现兄的情况 还没有做详细观察。但是, 这个句子也可用施 毫表原因。变化成 “我们养不出生丹来”。

需要讨论的是, 和前面五个例句同时举出的 还有一个例句。

57 爸, 这沟里的水, 不能排出去吗?

这个句子符合「不能 V R」使用的第一个条 件。句中的趋向动词 “出去” 和句中受事 “水” 可以构成一种陈述关系, 即 “水出去”。但是 句中没有施事出现, 根据前后文也补不出施事, 所以不符合「不能 V R」使用的第二个条件。

这种现象跟家问句有关。如果把上面的句子 变成陈述句，表达可能仍要用 $「 V$ 不 $R 」 。$

38 * 沟里的水不能排出去。

20沟里的水排不出去。

可是, 疑问句的情况就大不相同了, 「不能 $V R$ 」表示可能的两个条件都不具备，也可以 
用「不能V R」, 即「V 不 $R$ 」在是问句中都 可以用「不能V R」替换，也可以说表达可能 的「不能V R」在筑问句中几乎没有限制。下 面举几个有代表性的例子 :
6 下午五点, 你们不能打完球吗?
(11) 在东京你不能买上房子吗?
(2) 这次你们班不能打球吗?
63 这本书你不能看位吗?
(4) 黑板上的字不能徖掉吗?
(9) 他不能走回家去吗?
6 这座小你不能优上去吗?

不再多举例，已足以说明「不能 V R」在 问句中的使用情况。例@61是 A 类动结式 ; 例 663是B类动结式；例6和我们刚才讨论郡例 相同，虽是 C 类动结式，但句中没有施亭出 现；例66是A类动趋式。这些用例表明，「不 能V R」的使用在实问句中几乎不受限制。

2.「V不 $R 」$ 变换成「不能VR」后的两 种情况产生的原因

本文开头已经提到在「不能V R」不能代替 $\lceil V$ 不 $R$ 」表示可能意义的范围内，「V不R」 变换成「不能V R」后有两种情况。一种是变 块后句子就不成立了；另一种是变埃后句子仍 然成立，但 “不能” 表示㢣止的意义。这两种 情也可以说表示禁止的“不能”用在 “V R” 前面有两种俏况，即成立或不成立。诸看前面 的例句，这里不再重复。下面我目想讨一下 这两种情况形成的原因。

\section{1.「不能 VR」和「V不 $R$ 」的否定作} 用点不同

$\lceil V$ 不 $R 」$ 的 “不” 用在结果或㑇向之前, 否定的是某种结果或趋向实现的可能性, 不能 对动词发生作用, 所以它可以用在动作已经发 生之后。如 :

6) 黑板上的字怎么擦也搳不掉。

“腎么V也 V不 $\mathrm{R}$ ” 就表示动作虽已反变进 行, 但是结果或趋向仍不能实现。

与之相反,「不能 V R 」的意义作用点在动 词。“不能”可以无限制地用在动词前表示禁
止某一动作发生。如 :

68 这些菜不能卖。

如果动词后加上结果或趋向补语，以「V R」的形式出现, “不能”就不是对动作完全 禁止，而是对达到某种结果或趋向的动作的禁 此。如 :

9 这些莱不能买光。

例ほ中, “不能”是对 “实”这个动作的全 面等止；例6中，“不能”是对达到 “光”的 程度的“实”这个动作的菜止。

\section{2. “ $V R^{\prime}$ 结构的内部联系}

「VR」中的结果或趋向与动词的联系有两 种情况。

1. $V$ 可以控制 $R$ ，即动词可以制约后面的结 果或趋向。具体地说动作的结果或趋问能否实 现, 可以决定于动作是否进行到那种程度。 如：“卖光”“徖掉”“说出来”“站起来”等, “光”“掉” “出来” “起来”这些结果或趋向 能否实现，可以决定于 “买”“擦”“说”“站” 这些动作是否进行到那种程度。

2. $V$ 不能控制 $R$, 即动词不能制约其后面的 结果或趋向, 具体地说, 结果或趋向能否实现 不能由动作是否进行到某种程度来决定，而是 取决于与动作无关的某种倩况。如：“买到”

“搬动”“看出来”“想起来”等, “到” “动” “出来” “起来”这些结果或趋向能否实现, 不是由“买” “搬” “看” “想”所达到的程度 决定的。是否 “买到”要秙有没有实的; 是否 “激动” 要看有没有力气; 是否 “活出来”要 不有没有眼力, 是否“想起来”要雷记忆如 何。

2.3.「V不 $R 」$ 变换成「不能 VR」后的 两种情况产生的原因

由于 “不能” 的意义作用点在动词, 那么 “不能” 能否对整个「V R 」结构发生作用, 就取决于「VR」的内部联系。如果 $V$ 可以空 制 R, “不能”就可以对整个「VR」结构发 生作用。即禁止达到某种结果或趋向的动作发 生。如 :

(2) 黑板上的字復不掉。 
黑板上的字不能擦掉。

(1) 这些菜实不光。

这些莱不能卖光。

(2)病人站不起来。

病人不能站起来。

(3)吸烟的害处说不出来。

吸烟的害处不能说出来。

如果 V不能控制 R，那么 “不能” 就不能对

㱏个「VR」结构发生作用。这时,「V不R」 如果变换成「不能V R」句子就不成立了。 如 :

(4) 他买不到这个星期的机票。

* 他不能买到这个星期的机票。

(6) 老师的课我听不看楚。

*我不能听清楚老师的课。

(6) 那件件弶他想不起来了。

* 那件言他不能想起来了。

(1) 我看不出来小张有才能。

* 我不能看出来小张有才能。

“不能” 对「VR」的作用, 可以篔作是一 个连锁反映。“不能”的作用点在 V, 而 V又 能热制 $R$ ，所以“不能”的作用延及到 $R$; 反 之, $V$ 不能控制 $R$, “不能” 的作用到 $V$ 就中 断了。

从上面的分析我们可以看出：「VR」结构 的内部联系如何是决定「不能V R」是否成立 的关健。但是「VR」结构的内部联系还有一 些复杂情况，还有待于进一步探讨。例如：

在「VR」结构中, 某些结果或趋向是否被 动词制约, 有些是固定的。如 : “动” “到”不 被任何动词制约。有些是不固定的, 因动词而 㫒。如 :

(8) 吸烟的害处不能说出来。

* 小张的才能不能看出来。

90这里不能写上自己的名字。

*你不能买上新房子。

同是 “出来”, “说出来” 的 “出来” 可以受 “说”的制约，而“看出来”的“出来”不能 受“看”的制约。同是 “上”, “写上”里的 “上”被“写”制约, “买上”的“上”不被
“买”制约。所以前一句是成立的，后一句是 不能成立的。其中有的是受同一形式的不同意 义的影响, 有的还说不济。

在「V R 」结构中, 某一动词是否能制约某 一趋向或结果, 有些是固定的, 有些也是不四 定的，因语言环境的不同，有不同的表现。

80 山顶太冷，你不能爬上去。

*这座山太高，我不能爬上去。

8DO这个故本不能讲完, 留着明天再讲。

*吸烟的客处不能讲完。

以上两例，前一句 “ $\mathrm{V}$ ” 能制约 “ $R$ ”, 后 一句 “ $V$ ” 不能制约 “ $R$ ”。同一 $r \mathrm{VR}$ 」形 式 “ $V$ ” 能制约 “ $R$ ” 时, 「不能 $V R$ 」是成 立的, 反之, “ $V$ ” 不能制约 “ $R$ ” 时, 「不能 $V R$ 」是不成立的。

对这些问题还没有做深入细致的调查, 只好 留在以后继续考察。

\section{3. 结尾}

本文对「V不R」变换成「不能V R」后表 现出来的三种现象形成的原因和规律进行了考 察, 说到底取终解决的是「不能 V R」结构不 同用法的使用条件。但是由于研究不解深入， 本文所回答的问题也只是勾画出一个轮阔，一 些具体问题留待今后进一步考虑。

〈注〉这五例引自荒川清秀〈中国語の可能表 現一一/能 V R $/$ と $/ \mathrm{V}$ 得 $\mathrm{R} / \longrightarrow$, 引 用时略作改动。

\section{〈考支武〉}

吕权湘1980.〈现代汉语八百词〉商务印书馆 北京语言学院1982. 《现代汉语常用词语例解》 刘月华等1983. 〈实用现代汉语语法〉外语数 学与研究出版社

陆俭明等1990. (虚词〉人民教育出版社 刘月华1980. 〈可能补语用法的研究〉〈中国语 文〉第四期

服部昌文1970. 〈可能の能願動呞と可能補豆 の関連について〉〈北九州大学外国語紀要》 
第20号

杉村博文1979. 〈能学好・学得好・能学得好〉

〈日本語と中国語の対照研究〉226号

大内田三郎1979〈中国㶾の可能補語につい

て〉〈中国語学〉226号

大河内康蜜1980.〈助動詞と可能補語〉〈日本 語数育〉 41 号

小野秢樹1989. 〈中国語の可能表現一「他娌
性」を通しての「能V R」及び「V得 R」 の考察—〉〈中国語学〉237号

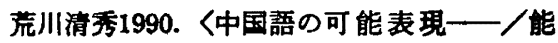
$\mathrm{V} \mathrm{R} /$ と $/ \mathrm{V}$ 得 $\mathrm{R} / \longrightarrow$, 学知大学〈外 語研紀要〉第14号

杉村文1991. 〈可能を表す助動詞と補語〉 〈中国語学習Q＆A〉大管館害店 